\title{
Improved double immunohistochemical staining method for cryostat and paraffin wax sections, combining alkaline phosphatase anti-alkaline phosphatase and indirect immunofluorescence
}

\begin{abstract}
Aims-To develop an immunohistochemical staining method for cryostat and paraffin wax sections so that two different antigens in the same section of tissues could be detected by combining immunoenzyme and immunofluorescence techniques.

Methods-This double immunohistochemical staining method combines alkaline phosphatase-anti-alkaline phosphatase (APAAP) using New Fuchsin as a chromogen and indirect immunofluorescence.

Results-APAAP staining for one antigen of this double immunohistochemical staining method was observed under bright field conditions alternating with immunofluorescence for another antigen under ultraviolet light. The double exposed photograph of both easily identified the two signals within the same cell. Conclusions-This double immunohistochemical staining method can overcome the disadvantages of any masking effect of the double immunoenzymatic methods and the background problems of double immunofluorescence method especially when applied to paraffin wax sections. It also permits good morphological identification of the doubly stained cells which may be of crucial importance in studies on pathology specimens.
\end{abstract}

(F Clin Pathol 1994;47:597-600)

The double immunohistochemical staining technique is used to demonstrate simultaneously the presence of two antigens (a) on two different cell types; (b) on different cells of the same type; and (c) on or inside the same cell. Technically, it is more difficult to perform than single immunostaining because of the need to avoid cross-reaction and any masking effect of signals between the two labelling systems. Several ways of performing double immunohistochemical staining have been used. Primary antibodies from different species, or primary antibodies of different immunoglobulin classes or subclasses from the same species can be used, followed by secondary antibodies to species specific or class or subclass specific immunoglobulins labelled with peroxidase and alkaline phosphatase. Use of directly conjugated antibodies with biotin or other ligands, followed by streptavidin or anti-ligand antibodies conjugated with peroxidase or alkaline phosphatase, is another option. Sequential double enzymatic labelling using primary antibodies from the same species was established when it was realised that the enzymatic reaction product of the first labelling system can effectively block its own antigenic sites. ${ }^{12}$ Either a combined peroxidase and alkaline phosphatase system or using the same enzyme system but different chromogens can be used for the detection step.

When the two antigens are located at the same subcellular site or are very close to each other-for example, membrane associated and cytoplasmic - it can be difficult to distinguish between the two enzymatic products by colour. Detection by double immunofluorescence can overcome this defect of detection by enzymatic reaction products, because detection of the signals uses separate filter systems and the two signals do not interfere with each other. However, this double immunofluorescence method is generally more suitable for studies using cell lines or cell smears which do not usually show serious background fluorescence, and is less applicable to tissue sections.

A double immunohistochemical staining method, combining immunoperoxidase staining and indirect immunofluorescence (FITC), has been described by Lechago et al in $1979 .{ }^{3}$ In this report we describe a double immunostaining system based on the same strategy but using an improved enzyme and detection system which can also work on paraffin wax sections.

\section{Methods}

Cryostat sections $(6 \mu \mathrm{m})$ were fixed in acetone for 10 minutes at room temperature, blocked with $10 \%$ normal rabbit serum and incubated with mouse monoclonal antibodies. Normal rabbit serum or irrelevant monoclonal antibody were used as negative controls. The slides were then stained using a standard alkaline phosphatase anti-alkaline phosphatase (APAAP) method. ${ }^{4}$ Rabbit antimouse immunoglobulin and APAAP complex (Dako Japan Ltd) were used at 1 in 50 and 1 in 
100 dilutions, respectively, at $37^{\circ} \mathrm{C}$ for 30 minutes and repeated once. New Fuchsin was used as a chromogen.

The New Fuchsin substrate solution was prepared fresh just before use by mixing solution I $(0.35 \mathrm{ml}$ of $5 \%$ New Fuchsin (Fluka 72200 , in $2 \mathrm{M} \mathrm{HCl}$ ) added to $0.35 \mathrm{ml}$ of fresh $4 \%$ sodium nitrite (Sigma S-2252) and agitated for 60 seconds) with solution II $(50 \mathrm{ml}$ of $0.05 \mathrm{M}$ TRIS $-1.2 \% \mathrm{NaCl}$ mixed with $18 \mathrm{ml}$ of 2-amino-2-methyl-1,3-propanediol (Merck 801464 ) and adjusted to $\mathrm{pH} 8.7$ to which 28 mg Levamisole (Sigma L-9756) was added) to which solution III (35 mg of naphthol ASBI phosphate (Sigma N-2250) dissolved in $0.42 \mathrm{ml} \mathrm{N}, \mathrm{N}$-dimethylformamide) was then added, and this mixture was used as the New Fuchsin substrate solution.

The slides were developed in this freshly prepared New Fuchsin substrate solution for 10 minutes at room temperature, then rinsed in TRIS-buffered saline $(0.05 \mathrm{M}$ TRIS- $0.15 \mathrm{M}$ $\mathrm{NaCl}, \mathrm{pH} 7 \cdot 6$ ), three times for three minutes each, and stained by indirect immunofluorescence method for the second marker (also using mouse monoclonal antibody). We found that acidic glycine elution ${ }^{5}$ is not necessary before the second step. After incubation with the second primary antibody FITC conjugated rabbit anti-mouse immunoglobulin antibodies ( $\mathrm{Fab}_{2}{ }_{2}$ fragment) (Dako Japan Ltd) were used at a 1 in 5 dilution for 30 minutes at $37^{\circ} \mathrm{C}$. Sections were then mounted with $2.5 \% \mathrm{DABCO} / 90 \%$ glycerol in TBS (pH 8.6) and kept at $4^{\circ} \mathrm{C}$ in darkness. No counterstaining was performed. With serial sections stained for the first marker by APAAP, unrelated antibody of the same isotype as the second primary antibody was used as a negative control in the second staining step. Positive cells were recorded as single and double exposure using a Nikon Optiphot microscope with appropriate filters and Kodak Professional Ektachrome EES 800/1600 film (set at ASA 800).

\section{Results}

The results of the double immunohistochemical staining of the cytospin of Raji cell line are shown in fig 1. APAAP staining for $B$ cell markers (L26 for CD20) revealed a red colour product on Raji cells when observed under bright field conditions (fig 1A); indirect immunofluorescence for Epstein-Barr virus latent membrane protein (LMP) showed a strong cytoplasmic and weak membrane apple-green fluorescence (fig 1C); and the double exposed photograph of both easily identified the two signals within the same cell (fig 1B). The weak cell surface membrane staining seen with antibodies to LMP is presumably due to some masking effect of this antigen by the alkaline phosphate reaction product. The results of double immunohistochemical staining identifying the lineage of the LMP positive cells in the cryostat (figs $2 \mathrm{~A}$ and B) and formalin fixed, paraffin wax sections (figs 2C and D) of an acute lymphoproliferative disorder induced by Epstein-Barr virus are shown in fig 2.
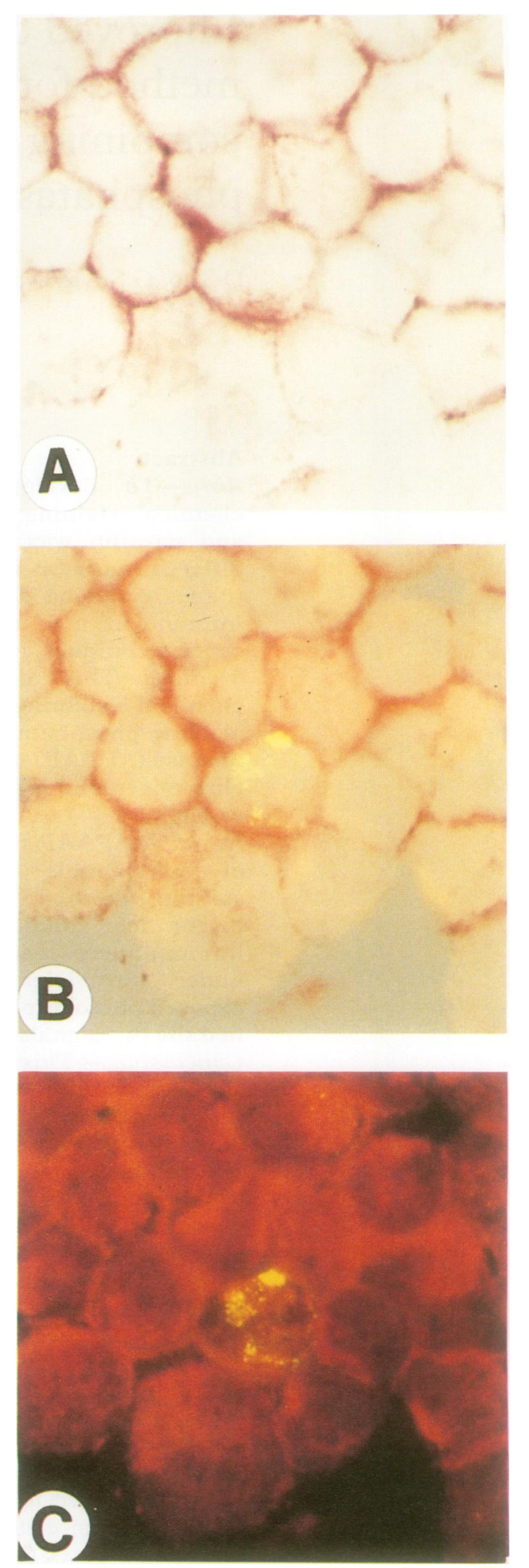

Figure 1 Double immunohistochemical staining showing Epstein-Barr virus latent membrane protein (LMP) positive cells in the cytospin preparation of Raji cell line. (A) APAAP staining for CD20, pictures taken under transmitted bright field illumination; (C) FITC immunofluorescence showing LMP; (B) double exposure of the left $(A)$ and right $(C)$ column pictures.

\section{Discussion}

We have successfully used this double immunohistochemical staining system to demonstrate the lineage of Epstein-Barr virus positive cells in lymphomas for one year. It 
Figure 2 Double immunohistochemical staining identifying the $T$ cell and non-T cell lineage of the LMP positive cells in the cryostat $(A, B)$ and formalin fixed, paraffin wax $(C, D)$ sections of an Epstein-Barr virus inducea acute lymphoproliferative disorder. Only double exposed pictures are shown. For $(A)$ and $(B)$ (arrow) $C D 3+L M P+$ cell $(A)$; $C D 3-L M P+$ cell $(B)$. Other cells are $C D 3+$ $L M P$-. For $(C)$ and $(D)$ (arrow):

$M T 1+L M P+\operatorname{cell}(C)$ $M T 1-L M P+$ cell $(D)$. Other cells are MT1 + LMP-
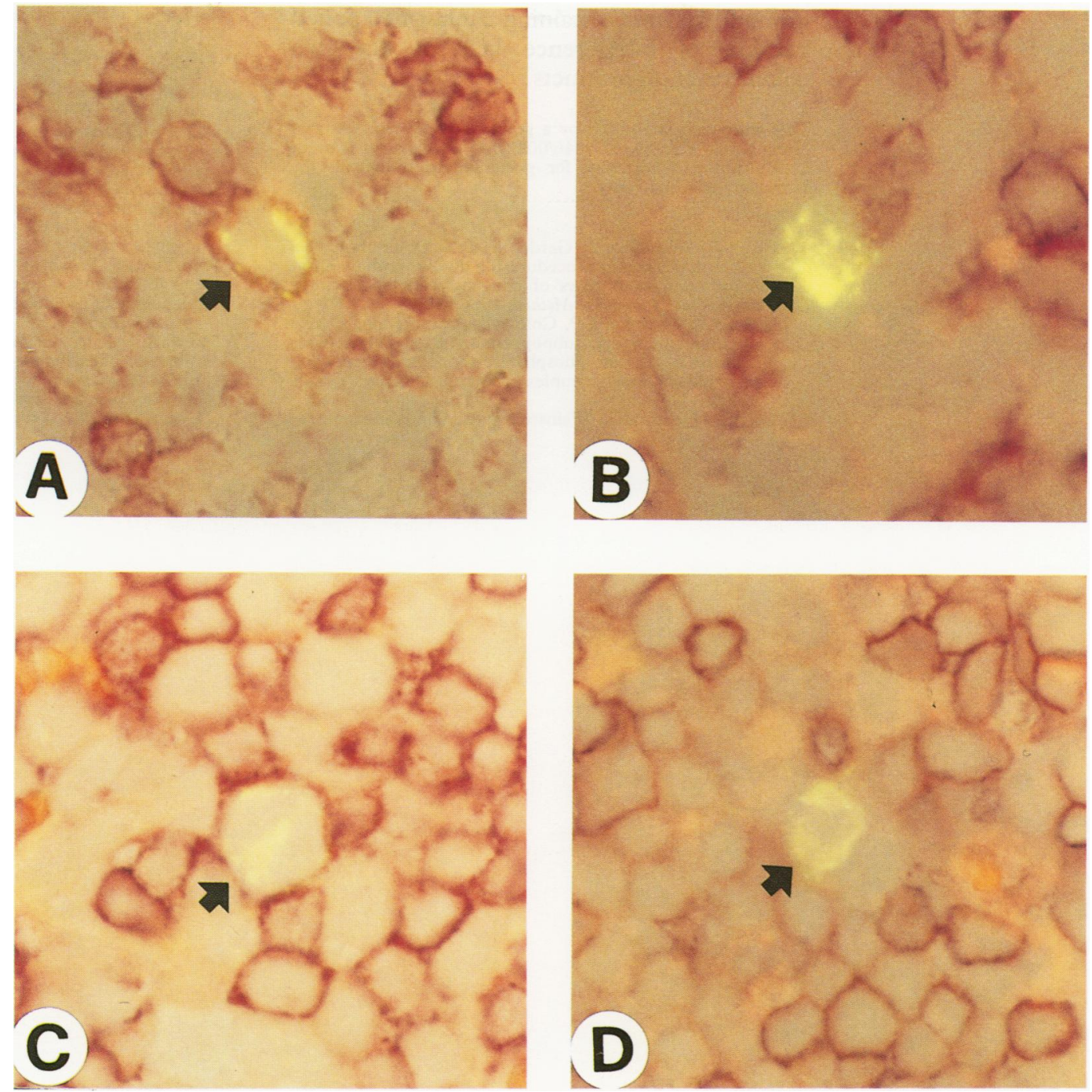

has given us constant and reliable results and enabled us to record the morphology of the labelled cells for detailed analysis. We have also successfully stained for cell markers and LMP in frozen sections of a lymph node biopsy specimen from a patient with acute Epstein-Barr virus infection and cases of lymphoma (data not shown) using this method. The pictures of the double exposure of the bright field and fluorescence microscopy easily located the two signals in the same cell. There was no cross-reaction between the two staining systems. Because the two signals were observed using different optical systems, there was no masking effect with each other. We have also successfully stained cryostat sections for $b c l-2$ oncoprotein (intracytoplasmic) and LMP (mainly cytoplasmic) using this method on a lymph node biopsy specimen from a patient with acute Epstein-Barr virus infection (results not shown).

Furthermore, we have extended our staining system to formalin and B5 fixed paraffin wax embedded sections. Because formalin and B5 fixed tissue sections usually show serious autofluorescence, double immunofluorescence can not be applied. However, in our double staining system, after the first APAAP staining, the enzymatic reaction confers on the whole section an evenly distributed weak red-colour background under ultraviolet light

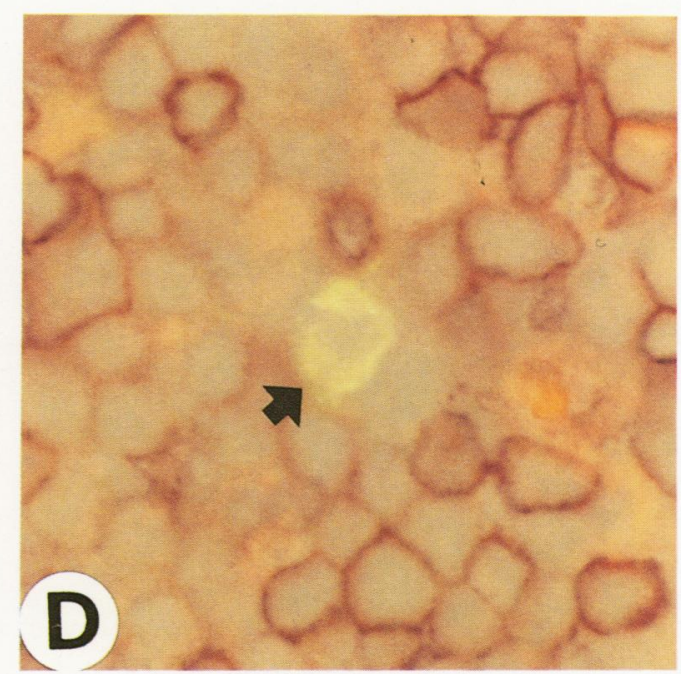

at the same excitation wavelength as for fluorescein isothiocyanate (FITC) fluorescence and the obvious apple-green colour of FITC stands out of the red background eliminating any problem created by autofluorescence of the section.

We found that the APAAP staining for cellular surface markers should be performed first, otherwise the enzymatic product of APAAP staining for cytoplasmic antigens quenches the antigenic sites of the cellular surface antigens. Ramshaw and Parums (1992) have also recently described similar strategy of double immunostaining, but using Fast Red TR salt as chromogen for APAAP. ${ }^{6}$ However, because the enzymatic products of Fast Red give strong red fluorescence under ultraviolet light, it can interfere with the FITC signals. ${ }^{7}$ We have also found that nitroblue tetrazolium (NBT) can be used as a chromogen in our system since it produces no background fluorescence as shown by New Fuchsin but the chemical reaction is more difficult to control than with New Fuchsin and has to be performed in darkness.

We recommend the use of this improved system for double immunostaining for work which requires a critical assessment of cell morphology to identify the type of cell labelled within the heterogeneous cell population of a tissue section, and where double 
immunoenzymatic staining cannot be applied because of interference between the two colour reaction products.

This study was supported by a grant from the University of This study was supported by a grant from the University of $\mathrm{Li} \mathrm{Ka}$ Shing Scholarship for postgraduate studies in the University of Hong Kong.

1 Fanili B, Abdulaziz Z, Gerdes J, et al. Description of a sequential staining procedure for double immunoenzymatic staining of pairs of antigens using mo

antibodies. F Immunol Methods 1986;93:265-73. different lymprman CP. Colour-contrast staining of two different lymphocyte subpopulations: a two-colour modification of alkaline phosphatase monoclonal anti-alkaline phosphatase complex technique. Stain Technol

3 Lechago J, Sun NCJ, Weinstein WM. Simultaneous visu- alization of two antigens in the same tissue section by combining immunoperoxidase with immunofluorescence techniques. I Histochem Cytochem 1979;27: 1221-5.

4 Cordell JL, Falini B, Erber WN, et al. Immunoenzymatic labelling of antibodies using immune complexes of alkaline phosphatase and monoclonal anti-alkaline phosphatase (APAAP complexes). $f$ Histochem Cytochem 1984;32:219-29.

5 Bologna M, Allen R, Dulbecco R. A method for double immunofluorescent staining by the indirect procedure with antibodies of the same isotypes. $\mathcal{F}$ Immunol Methods 1986;86:151-3.

6 Ramshaw AL, Parums DV. Combined immunohistochemical and immunofluorescence method to determine the phenotype of proliferating cell populations. F Clin Pathol 1992;45:1015-17.

7 Murdoch A, Jenkinson E, Johnson GD, Owen JJT Alkaline phosphatase-Fast Red, a new fluorescent label-Application in double labelling for cell surface antigen and cell cycle analysis. F Immunol Methods 1990;132:45-9. 